\title{
Perfil de los menores del Juzgado de la provincia de Castellón del 2012 al 2016
}

\section{Juveniles' profile in the Castellón province Court from 2012 to 2016}

Fecha de recepción: 19-07-2017

Fecha de aceptación: 9-11-2017

\author{
Keren Cuervo Gómez \\ Dpto. Psicología Evolutiva, Educativa, Social y Metodología. \\ Universitat Jaume I \\ Natalia Palanques Alegre \\ Dpto. Psicología Evolutiva, Educativa, Social y Metodología. Universitat Jaume I \\ Lidón Villanueva Badenes \\ Dpto. Psicología Evolutiva, Educativa, Social y Metodología. Universitat Jaume I \\ Rita López García \\ Coordinadora Equipo Técnico del Juzgado de Menores de Castellón. \\ Jesús M. López Castillo \\ Educador del Equipo Técnico del Juzgado de Menores de Castellón.
}

\section{resumen/alsstract:}

Diferentes sectores parecen ser partícipes de la creencia de que los menores suponen un gran peligro para la sociedad, y más aún si ya han formado parte de un proceso judicial. Esta percepción social se encuentra en gran medida influenciada por la alarma causada ante algunos delitos ampliamente publicitados por los medios de comunicación, que en ocasiones fragmentan y distorsionan los acontecimientos. Estas opiniones y demandas no pasan desapercibidas para el sistema penal, que ha intentado reflejar en el Código Penal español modificaciones encaminadas a proporcionar una mayor seguridad ciudadana. El objetivo de este estudio es el de establecer un perfil del menor con expediente en el Juzgado de Castellón, en cuanto a variables judiciales y sociodemográficas, con el fin de contrastar dichas creencias.

Los participantes de este estudio fueron todos los menores del Juzgado de la provincia de Castellón del año 2012 al 2016. Los resultados reflejan un perfil de menor mayoritariamente masculino, con una edad comprendida entre los 16 y los 17 años, que comete delitos de lesiones, robos con fuerza y hurtos, y representa al 1.6\% de la población general de menores. Estos datos apoyarían la tendencia no delictiva del menor en el Juzgado, ofreciendo un perfil mayoritario con un único expediente y un contacto puntual con el sistema judicial.

Different sectors seem to believe that minors suppose a great danger for the society and even more if they have been part of a judicial process. This social perception is greatly influenced by the alarm caused by some crimes widely publicized by the media, which sometimes fragment and distort the events. These opinions and demands do not go unnoticed for the penal system, which has tried to reflect some changes in the Spanish Penal Code in order to provide a greater citizen security. The aim of this study is to establish a profile of the minor of Castellón's Court, in terms of individual and judicial variables, in order to contrast these society beliefs.

The participants of this study were all the minors of the Castellón's province Court from 2012 to 2016. The results reflect a minor's profile mainly masculine, who is between 16 and 17 years old, who commit injury offenses, burglaries and thefts, and represents the $1.6 \%$ of minors' general population. These data highlight a non-delinquent juvenile profile in Cort, offering a majority profile with a single record and a punctual contact with the judicial system. 


\section{palabras clave/keywords:}

delitos, medidas educativas, reincidencia, delincuencia, menores infractores, estudio longitudinal.

crimes, educative measures, recidivism, delinquency, juvenile offenders, longitudinal study.

Existe un gran desconcierto ante las conductas delictivas cometidas por los menores de edad. Diferentes sectores parecen ser partícipes de la creencia de que los jóvenes en la actualidad suponen un gran peligro para la sociedad, y más aún si ya han formado parte de un proceso judicial. Parece que la sociedad percibe a los menores como más violentos y menos respetuosos que los de generaciones anteriores (Montero, 2008). Un 79.5\% de los encuestados en el estudio de Fernández y Tarancón (2010), en el que se evalúa la actitud de los ciudadanos hacia la respuesta al delito juvenil, considera que la delincuencia en los menores de edad ha aumentado, sobre todo los delitos violentos y los relacionados con las drogas. Se piensa que la mayoría de los delitos cometidos son violentos y se cree que la mayoría de los menores son reincidentes. De hecho, gran parte de los encuestados considera que entre el 26\% y más del 50\% de la delincuencia general cometida en España, es llevada a cabo por menores y que va en aumento (Fernández y Tarancón, 2010). De la misma forma, en un estudio realizado al respecto, la mayoría de los encuestados piensa que el nivel de reincidencia de los menores se sitúa entre el 50\% y el 75\% (García, Martín, Torbay y Rodríguez, 2010).

Por otra parte, parece que se considera el sistema de justicia como ineficaz y excesivamente indulgente con el infractor (Cullen, Fisher y Applegate, 2000; Hough y Roberts, 2004; Varona, 2008). Estas percepciones incluso se extrapolan a otros países anglosajones (Fernández y Tarancón, 2010; Hough y Roberts, 2004). Dichas creencias, podrían verse influenciadas en parte por la relativa impunidad ante los delitos cometidos por los menores de 14 años, inimputables ante la ley. El hecho de que no se les aplique el Código Penal, sino la Ley de Responsabilidad Penal del Menor, parece ser considerado como excesivamente benevolente y sin la severidad que se debería (Aizpurúa y Fernández, 2016; Fernández y Tarancón, 2010; Montero, 2008).

Al tratar factores específicos relacionados con la delincuencia en los menores de edad, los entrevistados consideran que las causas de la misma serían factores sociales y económicos $(87.8 \%)$, mientras que un grupo minoritario cree que sería producto de una decisión individual y racional de la persona (7.3\%) o de una deficiencia/enfermedad psíquica/adicción (4.9\%) (Fernández y Tarancón, 2010). Por otra parte, las formas consideradas como más adecuadas para reducir la delincuencia juvenil son el aumento de la disciplina en la familia y en la escuela (47.9\%), la imposición de condenas más duras (13\%) y otras estrategias menos punitivas como las de dar más oportunidades de empleo (13\%), más apoyo para los padres $(8 \%)$ o proporcionar más recursos para la escuela (6.7\%) (Fernández y Tarancón, 2010).

Esta percepción social de la delincuencia juvenil, se encuentra en gran medida influenciada por la alarma causada ante algunos delitos ampliamente publicitados por los medios 
de comunicación, que en ocasiones fragmentan y distorsionan los acontecimientos (Baz, Aizpurúa y Fernández, 2015; Bishop, 2006; Montero, 2008; Rodríguez et al., 2010). De hecho en el estudio de Fernández y Tarancón (2010), los encuestados informan de que estas percepciones provienen tanto de las noticias de prensa, televisión y radio (46.8\%), como de opiniones ciudadanas.

Estas apreciaciones y demandas no pasan desapercibidas para el Gobierno, que ha intentado reflejar en el Código Penal español algunas modificaciones encaminadas a proporcionar una mayor seguridad ciudadana (Baz et al., 2015; Varona, 2008). Estas tendencias no se dan únicamente en nuestro país, sino que son muchos los países que han aplicado reformas al sistema de justicia juvenil, afectando principalmente al tratamiento de los hechos delictivos más graves y de naturaleza violenta (Bishop, 2006; Mears, Hay, Gertz y Mancini, 2007).

Uno de los términos que se ha utilizado en los últimos tiempos para hacer referencia a la instrumentalización del derecho penal por parte de los gobernantes, es el de "populismo punitivo", el cual refleja las exigencias de la ciudadanía (Baz et al., 2015; Fernández y Tarancón, 2010). Sin embargo, a pesar de que resulta innegable la influencia de la opinión pública en la política criminal, parece ser que las reformas y modificaciones orientadas al endurecimiento de la intervención, no han satisfecho a la ciudadanía (Aizpurúa y Fernández, 2016; Garland, 2001; Hough, Jackson y Bradford, 2013; Varona, 2008, 2013).

No obstante, los estudios en los que se han analizado de forma específica los conocimientos de la ciudadanía en relación al sistema penal, han concluido que existe mucha desinformación y que la visión que se tiene del tratamiento del menor, tiende a ser exagerada y basada en prejuicios (Cullen et al., 2000; Fernández y Tarancón, 2010). Parece ser que la sociedad tiene una percepción magnificada de la delincuencia, considerando que es cuantitativa y cualitativamente más grave de lo que en realidad es (Baz et al., 2015). Estas opiniones contrastan con la realidad, ya que se responde en función de estereotipos y teniendo en mente los casos más graves y llamativos que transcienden a la opinión pública. Existen evidencias de que la ciudadanía no está bien informada en cuanto a cómo se aplican las leyes penales y cuál es su contundencia (Aizpurúa y Fernández, 2016; Fernández y Tarancón, 2010; Hough et al., 2013).

$\mathrm{Al}$ respecto, desde el Paradigma de la Criminología del Desarrollo, diferentes estudios demuestran que la mayoría de los jóvenes realiza algún tipo de actividad delictiva durante su adolescencia, pero solo algunos persisten en este tipo de conductas (Verbruggen, Van der Geest y Blokland, 2016). Muchos de los menores infractores analizados en diferentes estudios tanto en España como en el extranjero, cuentan en toda su trayectoria delictiva únicamente con un delito o expediente en el juzgado (Bravo, Sierra y del Valle, 2009; Capdevila, Ferrer y Luque, 2005; Cuervo, Villanueva y Pérez, 2017; Garrido, 2009; Iborra, Rodríguez, Serrano y Martínez, 2011; Jennings, 2002; San Juan y Ocáriz, 2009). La mayoría de carreras delictivas suelen ser abandonadas de manera natural y los menores que persisten en este tipo de conductas, suelen representar un pequeño número. Estos jóvenes presentan un inicio precoz en dichas conductas desadaptadas y cometen delitos más graves. De hecho, la información relativa a las memorias anuales de la Fiscalía General del Estado, no parece 
confirmar un aumento de los menores condenados. Esta afirmación se ve apoyada por el descenso en los escritos de alegaciones $(-10 \%)$ y las sentencias $(-5.56 \%)$ en las últimas memorias (Instituto Nacional de Estadística, 2016).

Después de presentar las percepciones que parece tener la ciudadanía acerca de los menores infractores, a continuación se muestran los rasgos característicos de esta población de jóvenes, tanto judiciales como sociodemográficos, en relación al tipo de delito, sexo, edad, nacionalidad, etc. Las detenciones de menores como presuntos autores de un delito o una falta penal, han descendido (Centro Reina Sofía y Ministerio del Interior, 2006). Sin embargo, también en los últimos años se ha constatado un incremento en los delitos violentos y contra la libertad sexual (Capdevila et al., 2005; Centro Reina Sofía y Ministerio del Interior, 2006) y en los actos violentos relacionados con el ámbito familiar y escolar. Además, ha habido un aumento de menores detenidos tanto por homicidio doloso y asesinato, como por lesiones (Centro Reina Sofía y Ministerio del Interior, 2006). Estos resultados parecen seguir tendencias analizadas en Europa, Canadá o Estados Unidos que muestran que no aumenta el nivel de delincuencia general, pero sí lo hacen algunos delitos violentos .

Por otra parte, al examinar el tipo de delito cometido en mayor medida por los menores, se encuentra que los resultados varían según la población estudiada. Varios estudios encuentran que el mayor porcentaje se sitúa en los delitos contra la propiedad (Capdevila et al., 2005; Garrido, 2009; Graña, Garrido y González, 2006), concretamente en robo con violencia o intimidación (San Juan y Ocáriz, 2009) y robo con fuerza en las cosas (Bravo et al., 2009). Y si este delito es cometido de forma violenta al inicio de las trayectorias delictivas, se asocia en mayor medida con la reincidencia (Ortega, García y Frías, 2014).

En relación a los rasgos sociodemográficos característicos de esta población de menores, se pueden encontrar datos coincidentes en los diferentes estudios. A continuación se destacan las principales tendencias. En cuanto al sexo, se observa que es mayoritariamente masculino, oscilando entre el 83\% y 87\% (Capdevila et al., 2005; Ministerio de Sanidad, Servicios Sociales e Igualdad, 2017). Aunque parece ser que la proporción de chicas detenidas ha ido aumentando en los últimos 10 años, tanto en esta comunidad como a nivel nacional (García, Zaldívar, Ortega, de la Fuente y Sáinz-Cantero, 2012).

La relación entre la edad y la criminalidad ha sido objeto de un gran número de estudios, e incluso es considerada como la variable que mejor explicaría la delincuencia (Smith, 1995). Parece ser que el volumen de los delitos cometidos se incrementa con la edad. La mayor cantidad de menores se sitúa en los 17 años, a partir de este punto las conductas delictivas tienden a disminuir (Alcázar, Bouso y Verdejo, 2015; Rechea y Fernández, 2006; San Juan y Ocáriz, 2009). Este efecto se podría explicar por la llamada "curva de edad", que defiende que la delincuencia suele declinar cuando la persona alcanza sus primeros años de madurez (Laub y Sampson, 2001). De hecho, el mayor porcentaje de delitos se sitúa en las edades mayores, dentro de la franja 16-17 (García et al., 2012; INE, 2017). La población extranjera en contacto con la justicia de menores, ha ido creciendo, al igual que lo ha hecho en todos los ámbitos de la vida social. La inmigración ha sido un fenómeno en alza en los últimos años y de contrastado impacto sobre la estructura demográfica y social de nuestro país (Ca- 
pdevila et al., 2005). Sin embargo, la mayoría de los menores de este colectivo continúan siendo españoles, seguidos de africanos, sudamericanos y resto de la unión europea (Alcázar et al., 2015; García et al., 2012; INE, 2017).

Son numerosos los estudios tanto es nuestro país como en el extranjero, que relacionan el consumo de alcohol y drogas con la comisión de delitos violentos e impulsivos. El alcohol es un factor incluso más vinculado a la delincuencia de menores que el resto de drogas. Es especialmente destacado el consumo en espacios festivos, ligado al ocio del fin de semana, y en condiciones de mayor desinhibición e impunidad por el anonimato del grupo o masa. Sin embargo, en la mayoría de los menores infractores no se apreciaría una relación significativa entre delincuencia y alcohol, exceptuando algunos colectivos pertenecientes a bandas subculturales violentas como los Skin Heads o los Latin Kings, o entre los menores extranjeros no acompañados. Solo un $7 \%$ de los menores infractores presentaría un consumo de drogas problemático, siendo el cannabis la droga más consumida (Alcázar et al., 2015).

En cuanto al ámbito familiar, un ambiente desestructurado, distante y coercitivo, puede producir conductas agresivas que relacionen al menor con la delincuencia (INE y Censo, 2011; Kinsworthy y Garza, 2010). Mientras que un ambiente familiar positivo con apoyo y afecto, ayuda al bienestar psicosocial del adolescente en gran medida. Sin embargo, cuando la familia se encuentra en etapa de transición, por rupturas, u otro tipo de circunstancias, esta estabilidad es difícil de mantener, y en algunos casos esto se relaciona con la delincuencia (Valgañoni, Muñoz y Briccola, 2014). A pesar de ello, dentro de los distintos tipos de composiciones familiares, la de tipo nuclear es la más representativa en los menores con medidas educativas en Madrid. El 10\% forman parte de familias desestructuradas, de ellas el $7 \%$ presentan ausencia del padre, el $2 \%$ ausencia de la madre y en el $1 \%$ de los casos son los servicios sociales quienes ostentan la tutela (Alcázar et al., 2015).

La situación formativa del menor, en general suele ser pobre. Según un estudio realizado en la Comunidad Valenciana, la mayoría no suelen estar escolarizados (68.4\%), teniendo en cuenta que alrededor de la mitad de los menores tienen más de 16 años y no están obligados a estarlo. El 80.09\% tiene un nivel bajo de hábitos y habilidades escolares. El 66.48\% ha sido absentista durante su etapa escolar o ha repetido algún curso (70\%) y el 66\% manifiesta un comportamiento negativo en el aula (Alcázar et al., 2015; Iborra et al., 2011).

Las medidas impuestas más comunes suelen ser la libertad vigilada, las prestaciones en beneficio a la comunidad y el internamiento semiabierto (Ministerio de Sanidad, Servicios Sociales e Igualdad, 2017). El Equipo Técnico de Toledo propone también en mayor medida la libertad vigilada, a la que sigue el archivo del expediente (Alcázar et al., 2015). Además, la Fiscalía General del Estado (2017) registró durante el año 2016 la medida de convivencia con otra persona, familia o grupo educativo como la tercera más impuesta.

\section{Objetivo}

Basándonos en todo lo anterior, con este estudio se pretende establecer un perfil descriptivo de los menores del Juzgado de Castellón, en cuanto a variables judiciales y sociodemográficas (proporción de menores que acuden al Juzgado, tipología de delitos, sexo, edad, etc.). 
El objetivo es el de contrastar la situación real de la provincia de Castellón, con las creencia sociales.

\section{Método}

\section{Participantes}

Los participantes de este estudio fueron todos los menores del Juzgado de la provincia de Castellón del año 2012 al 2016. El rango de edad de éstos osciló entre los 14 y los 17 años en el momento de comisión del delito. Los menores estudiados fueron en el año 2012: 321, en el 2013: 317, en el 2014: 426, en el 2015: 384 y en el 2016: 376, formando un total de $1824(\mathrm{X}=364.8)$ registros a lo largo de los 5 años. Los datos se corresponden con todos los menores infractores de la provincia, a los que el Ministerio Fiscal ha considerado abrir un expediente de reforma por estar implicados en alguna infracción penal.

\section{Procedimiento}

Los datos de este estudio se obtienen a partir del análisis de los expedientes de los menores en el Juzgado de Menores de Castellón. Son todos los datos registrados por el Equipo Técnico de la Fiscalía de Castellón del 2012 al 2016. Se analizó el número de expedientes de cada menor, la tipología de los delitos cometidos, la medida propuesta por el equipo técnico y algunos datos demográficos.

En este sentido, es necesario aclarar que el delito o falta que se ha contabilizado para este análisis es el que aparece en el decreto, y que en muchos casos no corresponde a la acusación judicial final, ya que tras la fase de instrucción, en la vista oral, puede demostrarse que se trata de delitos menos graves. En el caso de las medidas contabilizadas, se puntualiza que los análisis se refieren a las medidas propuestas por el Equipo Técnico, que pueden no corresponder en todos los casos a las medidas ejecutadas, aunque si lo suelen hacer en la mayoría de los casos (Alcázar et al., 2015).

\section{Resultados}

En la Figura 1 puede observarse la proporción de chicos y chicas atendidos en cada año (del 2012 al 2016). Teniendo en cuenta a todos los jóvenes con edades comprendidas entre los 14 y 17 años de la provincia de Castellón, el Equipo Técnico del Juzgado de Menores ha atendido en los últimos cinco años a una media del 1.6\% de dichos jóvenes. Parece que existe una estabilidad de alrededor del $1.5 \%$ de menores infractores por año, con picos que sobrepasan ese porcentaje en 2014 y 2016. Tal y como se aprecia en la Figura 1, el sexo de los menores infractores de la provincia de Castellón en los últimos cinco años, ha sido mayoritariamente masculino. Además, puede observarse que la proporción entre chicos y chicas se ha mantenido más o menos estable desde el año 2012 hasta el 2016. Como también se aprecia en la tabla 1 que el porcentaje directo de chicas se sitúa entre el $25 \%$ y el $30 \%$.

Figura 1. Proporción de menores atendidos por el Equipo Técnico de Castellón entre 2012 y 2016.

En relación a la edad, parece que los valores van oscilando a lo largo de los cinco años estudiados entre el intervalo de 14 a 15 y de 16 a 17 años, sin observarse una clara tendencia ha- 
Figura 1. Proporción de menores atendidos por el Equipo Técnico de Castellón entre 2012 y 2016.

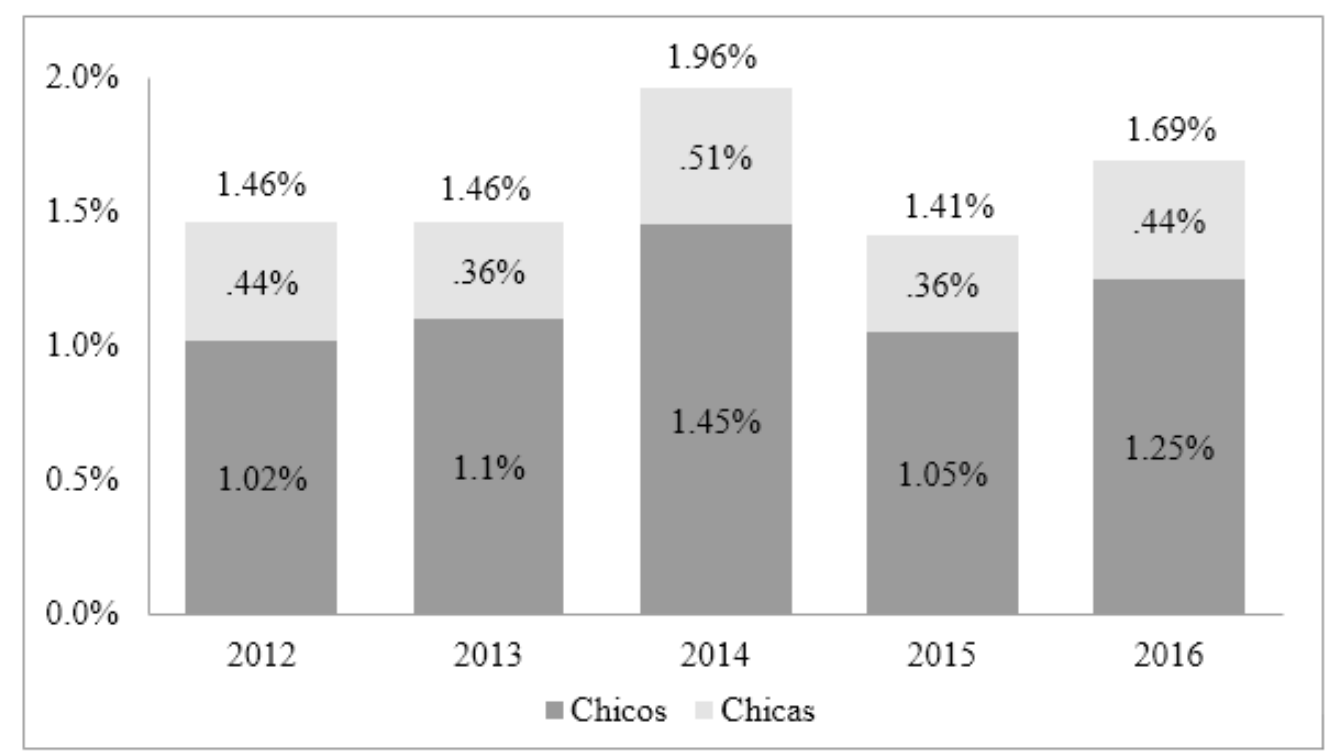

cia ninguna franja de edad (Tabla 1). Por lo que respecta a la nacionalidad, puede apreciarse en la Tabla 1 que el porcentaje mayoritario de menores atendidos en cada año corresponde a la nacionalidad española (alrededor del 70\%). Asimismo, parece observarse una ligera tendencia de aumento de menores infractores españoles y una disminución de extranjeros a lo largo de estos últimos cinco años. En cuanto a la estructura familiar, los porcentajes mayoritarios corresponden a las familias tradicionales, alrededor del $45-50 \%$, seguidas de las monoparentales y reconstituidas, ambas alrededor del 20\% (Tabla 1). En lo referente al área económica y de vivienda, se destaca que alrededor del $40 \%$ de las familias a las que pertenecen estos menores suelen presentar dificultades económicas.

Con respecto a la actividad formativo-laboral de los menores infractores de la provincia de Castellón, se aprecia que la mayoría de ellos estudia (alrededor del $60 \%$ cada año). A pesar de esto, parece que ha habido un ligero aumento en los últimos cinco años de jóvenes que se encuentran inactivos, es decir que ni estudian ni trabajan (Tabla 2). Profundizando en las problemáticas formativo-laborales, puede observarse que entre el $60 \%$ y $80 \%$ de los menores infractores presenta un bajo rendimiento académico y alrededor del $20 \%$ fracaso escolar.

En cuanto al consumo de tóxicos, coinciden los cinco años estudiados en que el tabaco es la sustancia más consumida entre los jóvenes infractores, aunque parece que ha habido un ligero decremento a lo largo de los años. Mientras que el consumo de hachís/marihuana y de alcohol, parece que ha aumentado progresivamente en estos últimos años. En el caso de la 
Tabla 1. Sexo, edad, nacionalidad y circunstancias familiares del año 2012 al 2016.

\begin{tabular}{|c|c|c|c|c|c|}
\hline & $\begin{array}{c}2012 \\
(\mathrm{~N}=321)\end{array}$ & $\begin{array}{c}2013 \\
(\mathrm{~N}=317)\end{array}$ & $\begin{array}{c}2014 \\
(\mathrm{~N}=426)\end{array}$ & $\begin{array}{c}2015 \\
(\mathrm{~N}=384)\end{array}$ & $\begin{array}{c}2016 \\
(N=376)\end{array}$ \\
\hline \multicolumn{6}{|l|}{ Sexo } \\
\hline - Chico & $70 \%$ & $75 \%$ & $74 \%$ & $74 \%$ & $74 \%$ \\
\hline - Chica & $30 \%$ & $25 \%$ & $26 \%$ & $26 \%$ & $26 \%$ \\
\hline \multicolumn{6}{|l|}{ Edad } \\
\hline - 14-15 años & $39 \%$ & $42 \%$ & $58 \%$ & $58 \%$ & $42 \%$ \\
\hline - 16-17 años & $62 \%$ & $58 \%$ & $42 \%$ & $42 \%$ & $58 \%$ \\
\hline \multicolumn{6}{|l|}{ Nacionalidad } \\
\hline - Español & $74 \%$ & $76 \%$ & $76 \%$ & $77 \%$ & $79 \%$ \\
\hline - Extranjero & $26 \%$ & $24 \%$ & $24 \%$ & $23 \%$ & $21 \%$ \\
\hline \multicolumn{6}{|l|}{ Estructura familiar } \\
\hline - Monoparental & $21 \%$ & $18 \%$ & $21 \%$ & $22 \%$ & $24 \%$ \\
\hline - Tradicional & $48 \%$ & $46 \%$ & $48 \%$ & $45 \%$ & $54 \%$ \\
\hline - Reconstituida & $20 \%$ & $22 \%$ & $20 \%$ & $20 \%$ & $16 \%$ \\
\hline - En proceso de cambio/separación & $4 \%$ & $4 \%$ & $4 \%$ & $4 \%$ & ------ \\
\hline - Convive con otras figuras & $6 \%$ & $8 \%$ & $6 \%$ & $6 \%$ & $6 \%$ \\
\hline - Con familia propia & $2 \%$ & $2 \%$ & ----- & ----- & ------ \\
\hline - Custodia compartida & ------ & ------ & $1 \%$ & $3 \%$ & ------ \\
\hline \multicolumn{6}{|l|}{ Área económica/vivienda } \\
\hline - Desempleo de los 2 progenitores & ----- & $22 \%$ & $21 \%$ & $19 \%$ & $17 \%$ \\
\hline - Dificultades económicas & $38 \%$ & $42 \%$ & $43 \%$ & $37 \%$ & $34 \%$ \\
\hline - Marginalidad/mendicidad & $10 \%$ & $7.8 \%$ & $5 \%$ & $7 \%$ & $10 \%$ \\
\hline - Impago hipoteca/alquiler & ----- & $7.5 \%$ & $5 \%$ & $6 \%$ & $7 \%$ \\
\hline - Reciben ayudas económicas & ----- & $25 \%$ & $23 \%$ & $25 \%$ & $25 \%$ \\
\hline $\begin{array}{l}\text { - Prestación por minusvalía de los } \\
\text { progenitores }\end{array}$ & ----- & $6.6 \%$ & $5 \%$ & $5 \%$ & $7 \%$ \\
\hline
\end{tabular}

reincidencia, parece que existe una estabilidad en la tasa de reincidencia que se sitúa alrededor del 30\% a lo largo de los años, con un ligero aumento del 34\% en el año 2015 (Tabla 2).

En cuanto a los delitos cometidos por los jóvenes infractores de la provincia de Castellón, puede apreciarse en la Figura 2 que son las lesiones, el robo con fuerza y el hurto los más cometidos en los últimos cinco años. Se destaca el hecho de que parece que existe un decremento del delito de robo con fuerza a lo largo de los años (23.76\% en 2012 al $13.51 \%$ en 
Tabla 2. Actividad formativa, consumo de tóxicos y reincidencia del año 2012 al 2016.

\begin{tabular}{|c|c|c|c|c|c|}
\hline & $\begin{array}{c}2012 \\
(\mathrm{~N}=321)\end{array}$ & $\begin{array}{c}2013 \\
(\mathrm{~N}=317)\end{array}$ & $\begin{array}{c}2014 \\
(\mathrm{~N}=426)\end{array}$ & $\begin{array}{c}2015 \\
(\mathrm{~N}=384)\end{array}$ & $\begin{array}{c}2016 \\
(\mathrm{~N}=376)\end{array}$ \\
\hline \multicolumn{6}{|l|}{ Actividad formativo/laboral } \\
\hline - Inactivo & $19 \%$ & $24 \%$ & $19 \%$ & $24 \%$ & $26 \%$ \\
\hline - Estudia & $62 \%$ & $67 \%$ & $67 \%$ & $64 \%$ & $63 \%$ \\
\hline - Trabaja & $5 \%$ & $1 \%$ & $3.5 \%$ & $4 \%$ & $4 \%$ \\
\hline - Desempleado & ----- & $19 \%$ & $12 \%$ & $3.5 \%$ & ----- \\
\hline \multicolumn{6}{|l|}{ Problemática formativo/laboral } \\
\hline - Bajo rendimiento académico & ----- & $83 \%$ & $57 \%$ & $73 \%$ & $70 \%$ \\
\hline - Fracaso escolar & ----- & $21 \%$ & $23 \%$ & $28 \%$ & $18 \%$ \\
\hline - Necesidades educativas especiales & ----- & $10 \%$ & $9 \%$ & $9 \%$ & $5 \%$ \\
\hline - Absentismo escolar & $42 \%$ & $45 \%$ & $28 \%$ & $39 \%$ & $38 \%$ \\
\hline - Expulsiones & $40 \%$ & $42 \%$ & $28 \%$ & $34 \%$ & $35 \%$ \\
\hline $\begin{array}{l}\text { - Problemas de relación con profe- } \\
\text { sores }\end{array}$ & ----- & $18 \%$ & $11 \%$ & $13 \%$ & $14 \%$ \\
\hline $\begin{array}{l}\text { - Problemas de relación con compa- } \\
\text { ñeros }\end{array}$ & ----- & $11 \%$ & $5 \%$ & $8 \%$ & $10 \%$ \\
\hline \multicolumn{6}{|l|}{ Reconocen consumo de tóxicos } \\
\hline - Tabaco & $48 \%$ & $44 \%$ & $41 \%$ & $47 \%$ & $42 \%$ \\
\hline - Alcohol & $33 \%$ & $32 \%$ & $35 \%$ & $37 \%$ & $48 \%$ \\
\hline - Hachís/Marihuana & $31 \%$ & $30 \%$ & $31 \%$ & $33 \%$ & $34 \%$ \\
\hline - Pastillas & $.62 \%$ & ----- & ----- & ----- & ----- \\
\hline - Cocaína & $.31 \%$ & $.9 \%$ & $.8 \%$ & $.5 \%$ & $.6 \%$ \\
\hline - Otros & $1 \%$ & $1.2 \%$ & $1.1 \%$ & $1.3 \%$ & $.86 \%$ \\
\hline Reincidencia & ----- & $27 \%$ & $28 \%$ & $34 \%$ & $26 \%$ \\
\hline
\end{tabular}

2016). La categoría de delitos contra la administración de justicia y otros, también incluiría los delitos contra el orden público, delitos contra la seguridad colectiva y falsedades. Especial mención merece el delito de maltrato familiar, que se sitúa prácticamente al mismo nivel de incidencia que el robo con violencia y los delitos contra el patrimonio (Figura 2).

Finalmente, en relación a las medidas educativas propuestas por el Equipo Técnico de Castellón, se aprecia en la Figura 3 que la mayor parte de las causas se archivan o se propone la libertad vigilada. Parece que a lo largo de estos últimos cinco años han ido disminuyendo las propuestas de prestaciones en beneficio a la comunidad y las conciliaciones, aunque forman parte de los grupos mayoritarios. Por el contrario, tienden a aumentar las tareas socioeducativas. Se destaca el escaso porcentaje de internamientos, que parecen reflejar un ligero descenso. 
Figura 2. Porcentajes de cada tipología delictiva del año 2012 al 2016.

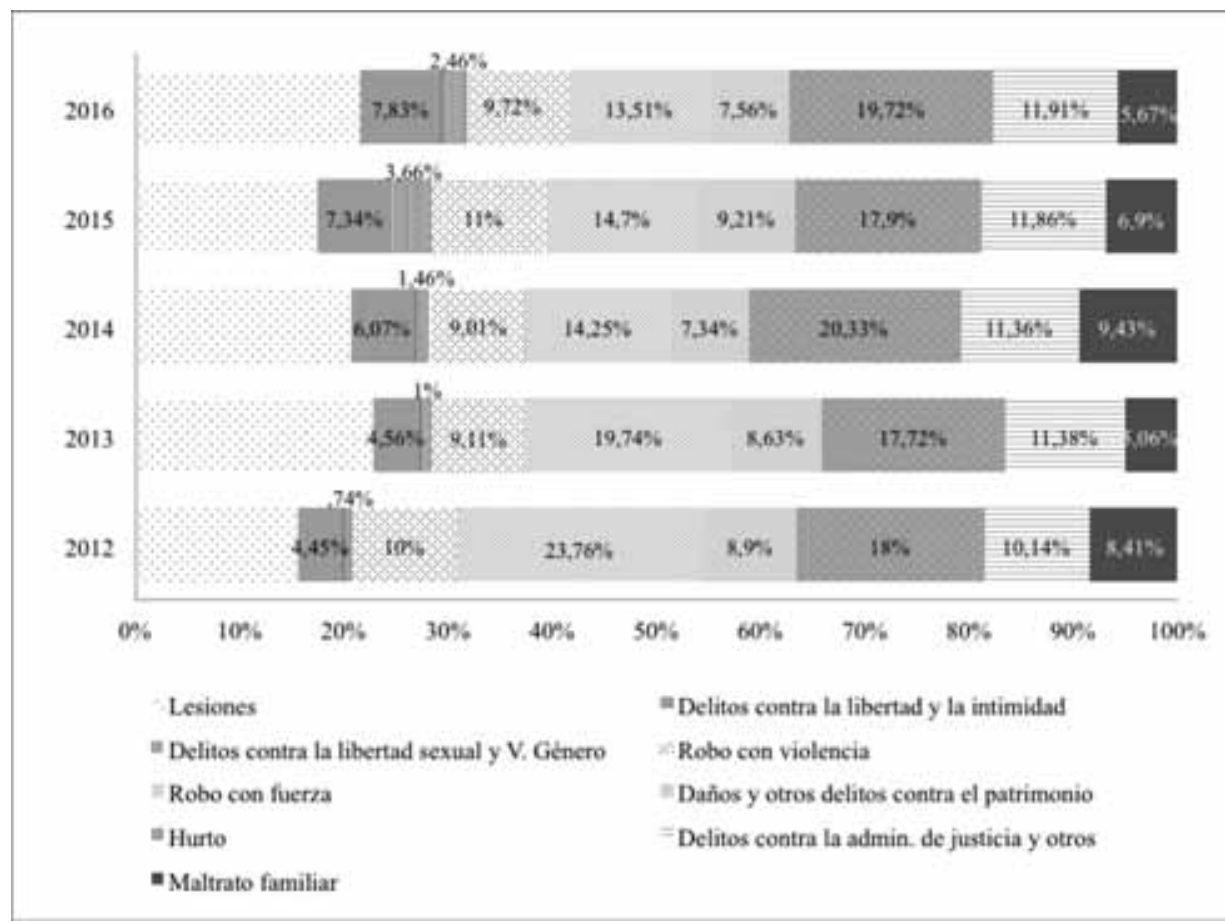

Figura 3. Medidas propuestas por el Equipo Técnico de Castellón del año 2012 al 2016.

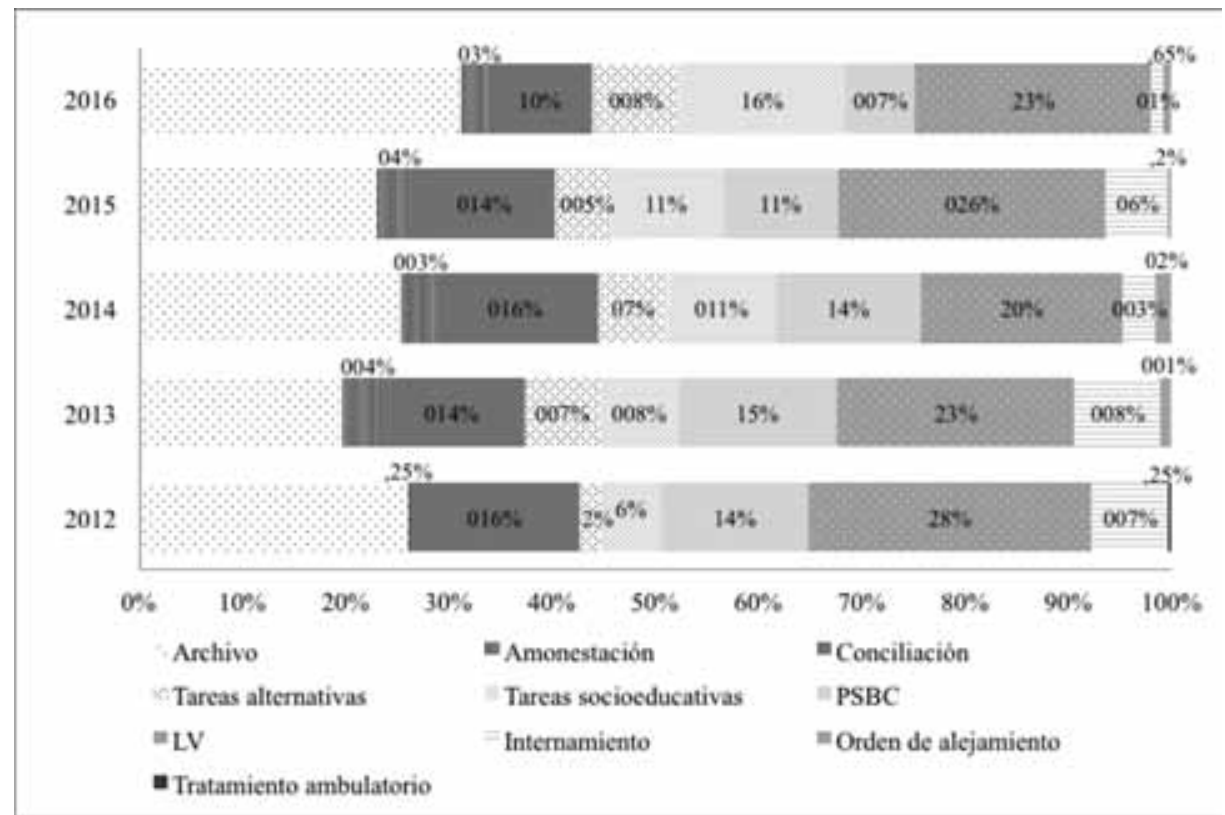

LV=Libertad Vigilada/ PSBC=Prestaciones Sociales en Beneficio de la Comunidad 


\section{Discusión}

El objetivo de este trabajo fue el de determinar un perfil real de los menores con expediente judicial en el Juzgado de la provincia de Castellón en los últimos 5 años, debido a que dichos menores son percibidos por la sociedad como más peligrosos de lo que realmente son (García et al., 2010). Un alto porcentaje de la ciudadanía cree que gran parte de la delincuencia está protagonizada por jóvenes. Sin embargo, con este estudio se comprueba que los adolescentes entre 14 y 17 años con algún expediente judicial, representan alrededor del $1.5 \%$ del total. La mayoría de estos menores, alrededor del 70\%, no reinciden. Este efecto se encuentra presente en diferentes estudios de nuestro país y del extranjero, en los que se confirma que con el asesoramiento e intervención de los integrantes de los sistemas de justicia, sería suficiente para que los menores no volvieran a cometer ningún tipo de delito (Alcázar et al., 2015; Bravo et al., 2009; Garrido, 2009; Iborra et al., 2011; San Juan y Ocáriz, 2009).

A continuación, se comentarán en primer lugar las variables sociodemográficas para seguir con las judiciales. Los resultados de este trabajo concluyen que el sexo de los menores ha sido mayoritariamente masculino, coincidiendo con otros estudios similares (Alcázar et al., 2015; Iborra et al., 2011). Aunque sí que parece haber un aumento de la presencia femenina entre este colectivo, ya que en otros estudios con datos del mismo Juzgado, el porcentaje ha sido menor (Capevila et al., 2005). En cuanto a la edad de los menores, la franja mayoritaria se sitúa entre los 16 y 17 años. Es decir, se encuentra un mayor número de menores entre 16 y 17 años que entre 14 y 15, coincidiendo con otros estudios en los que los delitos mayoritarios se dan en las edades más altas. En concreto, en el estudio de Alcázar et al. (2015) e Iborra et al. (2011) las edades en las que se registran más comisiones de delitos son los 16 y los 17 años, por lo que la percepción de alarma ante la impunidad de los menores con menor edad no estaría fundamentada. En cuanto a la nacionalidad de los menores, esta es mayoritariamente española, también coincidiendo con otros estudios, en los que alrededor del 70\% serían menores españoles (Alcázar et al., 2015; Iborra et al., 2011).

La estructura familiar más común entre los menores infractores es la de tipo tradicional. Alrededor de la mitad de ellos presenta una estructura en la que ambos progenitores estarían presentes en el núcleo familiar, seguidos de aquellos que forman parte de familias monoparentales y reconstituidas. Esta tipología familiar coincide con el estudio de Alcázar et al. (2015), en el que únicamente el 10\% de las familias son consideradas como desestructuradas.

Alrededor de la mitad de los menores del estudio consumen tabaco, seguidos de aquellos que consumen alcohol y hachís. En concreto, estos últimos parecen haber aumentado (Alcázar et al., 2015). En al ámbito escolar, más de la mitad de los menores se encontraría siguiendo algún tipo de estudio. Sin embargo, la mayoría tendría un bajo rendimiento académico y un $20 \%$ habría abandonado por completo los estudios sin obtener ninguna titulación.

Los delitos más comunes a lo largo de los últimos 5 años analizados, han sido las lesiones, el robo con fuerza y el hurto, sin gran oscilación entre los diferentes años. Estos resultados coinciden con el estudio de Alcázar et al. (2015), en el que se encuentra que los delitos más 
habituales son los robos, los daños y las lesiones. Estos hallazgos indicarían una mayor comisión de delitos de tipo instrumental, los cuales permiten a los menores la obtención de beneficios inmediatos. La comisión de este tipo de delitos es el medio para conseguir bienes necesitados o deseados, en casos en los que no se dispone de recursos. Mientras que en otros casos, es la obtención fácil, rápida y sin esfuerzo de dichos bienes, lo que lleva a los menores a la comisión de este tipo de actuaciones (Redondo, Martínez y Andrés, 2011). Llama la atención el hecho de que los porcentajes correspondientes al delito de maltrato familiar (en el que el mayor número de actos delictivos seguramente correspondan a violencia filio-parental), sean similares a los de los delitos más comunes como el robo con violencia o los delitos contra el patrimonio.

Las medidas propuestas que se han encontrado de forma mayoritaria en este estudio, han sido las de libertad vigilada, prestaciones en beneficio a la comunidad y conciliación, coincidiendo con otros estudios (Alcázar et al., 2015; Ministerio de Sanidad, Servicios Sociales e Igualdad, 2017). Al tener en cuenta la evolución a lo largo de los años, se comprueba que estas mismas medidas siguen siendo las mayoritarias, aunque sin observarse ninguna tendencia concreta, tal y como sucede en el estudio de Andalucía, en el que se analiza la evolución de las medidas del año 2007 al 2010 (García et al., 2012). Debe ser tenido en cuenta el porcentaje considerable de casos archivados, alrededor del 20-30\%, cuando se entiende que no es necesaria una mayor intervención, así como el elevado porcentaje de conciliaciones, alrededor del 10-15\%. A su vez, la proporción de internamientos es inferior al 10\%, de modo que más del $90 \%$ serían medidas propuestas en medio abierto. Estos datos refuerzan el perfil de bajo riesgo de los menores atendidos.

En general, la información de la que se dispone en nuestro país sobre la evolución y tendencia delictiva, es escasa. Debido a esa falta de datos, a los problemas metodológicos, a la imposibilidad de comparar los datos dependiendo de la forma de recogida de los mismos y a la "cifra negra", no se permite la adecuada recogida de datos oficiales (Fernández, Bartolomé, Rechea y Megias, 2009). En el estudio que nos ocupa, se recoge información real del Equipo Técnico de los últimos 5 años. Sin embargo, no se puede determinar si las oscilaciones de año son significativas, por lo tanto se describen únicamente tendencias generales.

Por consiguiente, como puede concluirse de los datos anteriores, y a pesar de las creencias tanto de nuestro país como de otros, estos resultados rebaten la peligrosidad de los menores infractores, ofreciendo un perfil mayoritario de sexo masculino, con una edad comprendida entre los 16 y 17 años y de nacionalidad española. En relación a los aspectos judiciales, este menor tendría un único expediente, un contacto puntual con el sistema judicial y habría cometido principalmente delitos contra la propiedad, coincidiendo con otros estudios (Hough y Roberts, 2004; Varona, 2008).

Así pues, se destaca el perfil no delictivo del menor con expediente judicial en el Juzgado de Menores, teniendo en cuenta que de todos los adolescentes con edades comprendidas entre los 14 y los 18 años, únicamente alrededor del 1.5\% entraría en contacto con la justicia. Por lo que parece ser que la concepción general que tienen los ciudadanos sobre la justicia, es poco eficaz y laxa, como se ha comentado anteriormente. Sin embargo, cuando se pregunta 
a la población por la intervención que ellos impondrían en un caso determinado, (mediante el uso de casos-escenario o supuestos en cuanto a edad del menor, reincidencia o delito concreto), las decisiones de los ciudadanos no difieren significativamente de las proporcionadas por los jueces, llegando a ser incluso más indulgentes que éstas (Baz et al., 2015). Parece ser que la información concreta del menor, promueve una tendencia hacia condenas menos punitivas y más rehabilitadoras y educativas, siempre que no se trate de delitos violentos (Cullen et al., 2000; Varona, 2008). De modo que ante estas concepciones, se debería atender con un mayor criterio y objetividad el sentir de la opinión pública a la hora de dar legitimidad a las reformas legislativas (Fernández y Tarancón, 2010).

Como argumentan algunos autores, en nuestra sociedad existiría un miedo al menor, en lugar de una preocupación por el mismo y tenderíamos a protegernos de éste, en lugar de intentar actuar desde la prevención e intervención teniendo en cuenta las circunstancias vitales que hayan podido influir a la hora de cometer la conducta delictiva (Cabezas, 2012). El delito no sería más que un fenómeno episódico y transitorio en la vida de gran parte de los menores infractores, por lo que según Montero (2008, p.3) "criminalizar a la juventud no es el camino y tampoco la solución".

Estos menores componen un sector importante de la población por su vulnerabilidad, debiendo utilizarse todos los medios disponibles para cubrir sus necesidades evitando que reincidan en estos comportamientos delictivos. Como proponen Alcázar et al. (2015), sería interesante contar con un registro territorial que recogiera de forma sistematizada las diferentes variables tanto sociodemográficas como judiciales de estos menores, con el fin de analizar las variaciones a lo largo de los años y comparar la información de las distintas provincias. De esta forma, al detectar con exactitud cuáles son las variables o factores que les conducen o influyen a la hora de cometer delitos, se estarían evitando futuros comportamientos delictivos.

\section{Referencias}

Aizpurúa, E.; Fernández, E. (2016). Opinión pública hacia el castigo de los delincuentes: La importancia del delito cometido. Boletín Criminológico, 1(161), 1-6.

Alcázar, M.A.; Bouso, J.C.; Verdejo, A. (2015). Análisis descriptivo de la actividad del Equipo Técnico de la Fiscalía de Menores de Toledo. Años 2001 al 2012. Anuario de Psicología Jurídica, 25(1), 97-106.

Baz, 0.; Aizpurúa, E.; Fernández, E. (2015). Factores explicativos de las actitudes hacia el castigo juvenil. Evidencias de un diseño factorial. Política y Sociedad, 52(3), 869-895.

Bishop, D.M. (2006). Public opinion and juvenile justice policy: Myths and misconceptions. Criminology \& Public Policy, 5(4), 653-664.Principio del formularioFinal del formulario

Bravo, A.; Sierra, Mã.; del Valle, J.F. (2009). Evaluación de resultados de la ley de responsabilidad penal de menores. Reincidencia y factores asociados. Psicothema, 21(4), 615-621.

Cabezas, J. (2012). Protegernos de los jóvenes. Revista Crítica Penal y Poder, (2), 189-200.

Capdevila, M.; Ferrer, M.; Luque, E. (2005). La reincidencia en el delito en la justicia de menores. Documentos de trabajo. Área Social y Criminológica del departamento de justicia. Barcelona: Generalitat de Catalunya. 
Centro Reina Sofía y Ministerio del Interior (2007). Delincuencia juvenil. Estadísticas del Centro Reina Sofía. Gobierno de España. Recuperado de http://www.centroreinasofia.es

Cuervo, K.; Villanueva, L.; Pérez, J. M. (2017). Riesgo de reincidencia y evolución, a través del inventario IGI-J en una población de menores infractores. Revista Internacional de Sociología, 75(2), 1-11.

Cullen, F.T.; Fisher, B.S.; Applegate, B.K. (2000). Public opinion about punishment and corrections. Crime and Justice, 27, 1-79.

Fernández, E.; Bartolomé, R.; Rechea, C.; Megías, A. (2009). Evolución y tendencias de la delincuencia juvenil en España. Revista española de Investigación Criminológica, 7, 1-29.

Fernández, E.; Tarancón, P. (2010). Populismo punitivo y delincuencia juvenil: Mito o realidad. Revista Electrónica de Ciencia Penal y Criminología, 12(8), 1-25.

Fiscalía General del Estado (2017). Memoria anual del año 2016: Menores. Madrid: Fiscalía General del Estado.

García, Ma D.; Martín, E.; Torbay A.; Rodríguez C. (2010). La valoración social de la Ley de Responsabilidad Penal de los Menores. Psicothema, 22(4), 865-871.

García, J.; Zaldívar, F.; Ortega, E.; de la Fuente, L.; Sáinz-Cantero, B. (2012). Justicia juvenil en Andalucía. Diez años de funcionamiento de la ley orgánica de responsabilidad penal del menor. Consejería de Justicia e Interior. Junta de Andalucía.

Garland, D. (2001). The culture of control. Crime and social order in contemporary society. Oxford: Oxford University Press.

Garrido, V. (2009). La predicción y la intervención con los menores infractores: Un estudio en Cantabria. (Estadísticas, instrumentos, protocolos y evaluación). Gobierno de Cantabria, Consejería de Empleo y Bienestar Social. Cantabria: Dirección General de Políticas Sociales.

Graña, J.L.; Garrido, V.; González, L. (2006). Reincidencia delictiva en menores infractores de la Comunidad de Madrid: Evaluación, características y propuestas de intervención. Madrid: Agencia para la Reeducación y Reinserción del Menor Infractor (A.R.R.M.I.).

Hough, J. M.; Roberts, J. V. (2004). Youth crime and youth justice: Public opinion in

England and Wales. Bristol: Policy Press.

Hough, M.; Jackson, J.; Bradford, B. (2013). Legitimacy, trust and compliance: An empirical test for procedural justice theory using the European Social Survey. En J. Tankebe; A. Liebling (Eds.) Legitimacy and criminal justice: An international exploration. Oxford: Oxford University Press.

Iborra, I.; Rodríguez, A.; Serrano, A.; Martínez, P. (2011). Situación del menor en la Comunitat Valenciana: Víctima e infractor. Valencian International University y Centro Reina Sofía. Valencia: Generalitat Valenciana.

Instituto Nacional de Estadística, INE (2017). Estadística de condenados: Adultos/ Estadística de condenados: Menores. Madrid: Instituto Nacional de Estadística.

Instituto Nacional de Estadísticas y Censos (2011). Encuesta de victimización y percepción de inseguridad 2011. Disponible en http://www.ecuadorencifras.gob.ec/encuesta-de-victimizacion-y-percepcion-de-inseguridad-2011/

Jennings, D. (2002). One year juvenile reconviction rates. July 2000 cohort. London: Home Office National Statistics.

Kinsworthy, S.; Garza, Y. (2010). Filial therapy with victims of family violence: A phenomenological study. Journal of Family Violence, 25(4), 423-429.

Laub, J.H.; Sampson, R.J. (2001). Understanding desistance from crime. Crime and Justice, 28, 1-69.

Mears, D.P.; Hay, C.; Gertz, M.; Mancini, C. (2007). Public opinion and the foundation of the juvenile court. Criminology, 45(1), 223-257.

Ministerio de Sanidad, Servicios Sociales e Igualdad (2017). Boletín de datos estadísticos de medidas impuestas a menores infractores. Boletín número 15. Datos 2015. Madrid: Ministerio de Sanidad, Servicios Sociales e Igualdad, Centro de publicaciones. 
Montero, T. (2008). La política criminal juvenil en España y el síndrome de Cristóbal Colón. Diario La Ley, 6919, 1-6.

Ortega, E.; García, J.; Frías, M. (2014). Meta-análisis de la reincidencia criminal en menores: Estudio de la investigación española. Revista Mexicana de Psicología, 31(2), 111-123.

Rechea, C.; Fernández, E. (2006). Continuity and change in the Spanish Juvenile Justice System. En J. Junger-Tass; S. Decker (Eds.). Handbook of Juvenile Justice (pp. 325-350). Holland: Kluwer/Springer.

Redondo, S.; Martínez, A.; Andrés, A. (2011). Factores de éxito asociados a los programas de intervención con menores infractores. Serie Informes, Estudios e Investigación. Madrid: Ministerio de Sanidad, Política Social e Igualdad.

San Juan, C.; Ocáriz, E. (2009). Evaluación de la intervención educativa y análisis de la reincidencia en la Justicia de Menores en la CAPV. Vitoria-Gasteiz: Servicio Central de Publicaciones del Gobierto Vasco.

Varona, D. (2008). Opinión pública y justicia penal: Resultados de un estudio piloto I. Boletín Criminológico, $103,1-4$.

Verbruggen, J.; Van der Geest, V. R.; Blokland, A. A. (2016). Adult life adjustment of vulnerable youths. The relationship between criminal history, employment history and adult life outcomes. Journal of Developmental and Life-Course Criminology, 2(4), 466-493. 